\title{
A CLOSE-PAIR ANALYSIS OF DAMP MERGERS AT INTERMEDIATE REDSHIFTS
}

\author{
Richard C. Y. Chou ${ }^{1}$, Carrie R. Bridge ${ }^{2}$, AND Roberto G. Abraham ${ }^{1}$ \\ ${ }^{1}$ Department of Astronomy and Astrophysics, University of Toronto, 50 St. George Street, Toronto, \\ ON M5S 3H4, Canada; chou@astro.utoronto.ca, abraham@astro.utoronto.ca \\ 2 Department of Astronomy, California Institute of Technology, 1200 East California Boulevard, Pasadena, \\ CA 91125, USA; bridge@ astro.caltech.edu \\ Received 2012 April 24; accepted 2012 October 9; published 2012 November 14
}

\begin{abstract}
We have studied the kinematics of $\sim 2800$ candidate close-pair galaxies at $0.1<z<1.2$ identified from the Canada-France-Hawaii Telescope Legacy Survey fields. Spectra of these systems were obtained using spectrometers on the $6.5 \mathrm{~m}$ Magellan and $5 \mathrm{~m}$ Hale telescopes. These data allow us to constrain the rate of dry mergers at intermediate redshifts and to test the "hot halo" model for quenching of star formation. Using virial radii estimated from the correlation between dynamical and stellar masses published by Leauthaud et al., we find that around $1 / 5$ of our candidate pairs are likely to share a common dark matter halo (our metric for close physical association). These pairs are divided into red-red, blue-red, and blue-blue systems using the rest-frame colors classification method introduced in Chou et al.. Galaxies classified as red in our sample have very low star formation rates, but they need not be totally quiescent, and hence we refer to them as "damp," rather than "dry," systems. After correcting for known selection effects, the fraction of blue-blue pairs is significantly greater than that of red-red and blue-red pairs. Red-red pairs are almost entirely absent from our sample, suggesting that damp mergers are rare at $z \sim 0.5$. Our data support models with a short merging timescale $(<0.5 \mathrm{Gyr})$ in which star formation is enhanced in the early phase of mergers, but quenched in the late phase. Hot halo models may explain this behavior, but only if virial shocks that heat gas are inefficient until major mergers are nearly complete.
\end{abstract}

Key words: galaxies: evolution - galaxies: formation - galaxies: interactions

Online-only material: color figures

\section{INTRODUCTION}

The mass assembly history of the universe is a major observable predicted by galaxy formation and evolution scenarios. Observational data have shown that galaxies in the local universe generally fall into two categories: quiescent ellipticals, which dominate the massive galaxy population $\left(M_{*} \geqslant\right.$ $\left.10^{10.5} M_{\odot}\right)$, and blue star-forming disk galaxies, which occupy the lower mass regime (Baldry et al. 2004; Balogh et al. 2004; Kauffmann et al. 2003). This color bimodality, which groups galaxies into the "blue cloud" and "red sequence," exists not only in the local universe, but also out to redshift $z \sim 1$ (Bell et al. 2004; Willmer et al. 2006), and possibly up to $z=2$ and beyond (Pozzetti et al. 2010; Conselice et al. 2011). The origin and the evolution of this color bimodality are largely unknown. The traditional view of red systems is that they are early-type galaxies that form monolithically at high redshift (Eggen et al. 1962), although in modern theories of galaxy evolution "nurture" plays as big a role as "nature," and evolution is driven by several physical mechanisms, including galaxy-galaxy interactions (Blumenthal et al. 1984; Di Matteo et al. 2005; Hopkins et al. 2010; Lambas et al. 2012), in-falling cold gas (Dekel \& Birnboim 2006; Dekel et al. 2009; Kereš et al. 2009; Bournaud et al. 2011), and hot halo gas quenching (Birnboim \& Dekel 2003; Birnboim et al. 2007; Panuzzo et al. 2011; Gabor \& Davé 2012).

There is now a considerable body of evidence suggesting that the red sequence is populated by galaxies that have somehow "migrated" there from the blue cloud (Martin et al. 2007; Hughes \& Cortese 2009; Bundy et al. 2010; Shapiro et al. 2010). Observations have shown that the total stellar mass of red massive galaxies $\left(M_{*} \geqslant 10^{11} M_{\odot}\right)$ has grown by a factor of two since redshift $z \sim 1$, while that of blue disk galaxies remains more or less the same (Bell et al. 2004; Faber et al. 2007; Abraham et al. 2007). Furthermore, Brammer et al. (2011) show that this mass growth trend can be traced up to $z \sim 2.2$. There is some evidence suggesting that the (visible wavelength) color transformation timescale for migration of a blue galaxy onto the red sequence is fast, $\sim 1 \mathrm{Gyr}$ (Bell et al. 2004; Blanton 2006). Assuming that the average gas to total mass ratio of late-type galaxies is $\sim 15 \%$ (Rubin et al. 1985) and a typical constant star formation rate is a few solar mass per year, it is difficult to consume all the gas content in blue galaxies within a timescale of 1 Gyr. In addition, simulations show that filaments in the intergalactic medium can also channel new and fresh gas into galactic halos, continuously feeding galaxies (Brooks et al. 2009; Kereš et al. 2009). Thus, in order to move a galaxy from the blue cloud onto the red sequence, the star formation must be sharply cut off or quenched, rather than being stopped simply by a depleted supply of cold gas. Most mechanisms invoked for quenching star formation involve various forms of feedback which couple star formation in disks to hot gas in galactic halos (Gabor et al. 2010).

One well-studied mechanism for halting star formation is the hot halo quenching model, which suggests that as gas falls into galactic dark matter halos it is heated by virial shocks. If the mass of a dark matter halo exceeds $\sim 10^{12} M_{\odot}$, then the rate of gravitational heating by shocks is greater than that of radiative cooling. As a result, a hot virialized gas halo is formed near the virial radius. These hot halo shocks heat up the in-falling cold gas immediately, which prevents the gas from collapsing and forming new stars (Birnboim \& Dekel 2003; Birnboim et al. 2007). The hot halo quenching model alone cannot quench all star formation within a galaxy since some in-falling gas will still cool down and form stars in the center of the galaxy, but this model provides a simple explanation for the mass-dependent 
bimodal galaxy distribution (Dekel \& Birnboim 2006; Cattaneo et al. 2006; Panuzzo et al. 2011).

Another interesting mechanism for halting star formation in galaxies is major merger quenching. In this picture, intense star formation triggered by the merging of two or more galaxies of roughly comparable stellar mass produces strong stellar winds that expel and/or heat the interstellar medium (ISM) through shocks or feedback from supernovae (Cox et al. 2006; Ceverino \& Klypin 2009). Stellar feedback from supernovae is probably not sufficient to quench the star formation of the entire galaxy (Springel et al. 2005), so some additional energy source is probably required to supplement this quenching process.

Some semi-analytical models integrate these various ideas into a hybrid picture in which a central super massive black hole $(\mathrm{SMBH})$ is an additional energy supply. In the hot halo quenching model, the in-falling cold gas could accrete on to the SMBH and trigger low-luminosity active galactic nucleus (AGN) and radio emission, which is also known as the "radio" mode of the quenching process (Croton et al. 2006; Hopkins \& Hernquist 2006). Similarly, major mergers can induce more violent material accretion and generate large amounts of energy feedback, which is known as the "quasar" mode of the quenching process. Both of these energetic feedback mechanisms involving the central SMBH could effectively expel or heat the surrounding gas disk and further quench the star formation activities (Hopkins et al. 2006).

Irrespective of how quenching operates, galaxy mergers are the central building blocks of the standard $\Lambda$-dominated cold dark matter model (Cole et al. 2008; Neistein \& Dekel 2008). In this model, major mergers transform disk galaxies into more massive spheroids (Toomre \& Toomre 1972; Toomre 1977). However, it is difficult to study the full process of star formation and/or quenching mechanisms from single snapshots of galaxy mergers. An alternative way forward is to study the evolution of large samples of mergers as a function of redshift and to connect these observations to models for triggering or quenching star formation. One approach along these lines is to investigate the merger rate density evolution of "wet" and "dry" mergers at different merging stages. The so-called wet mergers refer to galaxy mergers with intense star formation, while dry mergers are those with weak star formation.

Recent studies suggest that the fraction of dry mergers is low at high redshifts $(z \geqslant 0.5)$ but that dry mergers become important at low redshifts $(z<0.2$; Lin et al. 2008; de Ravel et al. 2009; De Propris et al. 2010; Chou et al. 2011). However, the reason for this change in the dominant mode of merging, the mechanism by which color transformations occur, and the corresponding merging timescales remain uncertain. A significant complication is that different procedures for defining samples result in mergers with quite different properties. In essence, there are two main ways to define samples of mergers. The first is based on morphological criteria and focuses on structural disturbances and/or tidal features (Conselice et al. 2000, 2003; Bridge et al. 2007, 2010; Jogee et al. 2009; Chou et al. 2011). Because mergers tend to show strong morphological disturbances at the first encounter and the last merging stage (Conselice 2006; Lotz et al. 2008), morphological selection methods are biased toward selecting mergers at later merging stages. The second approach is based on dynamical selection, which identifies mergers by limiting the projected physical separation and the velocity differences between two close-pair members (Patton et al. 2000; Lin et al. 2004a, 2008; LópezSanjuan et al. 2010).
Bridge et al. (2010) showed that when these different merger selections were normalized to the timescale in which they were sensitive to identifying mergers, the majority of studies agreed that the merger rate increases with redshift; however, there remain some discrepancies in the absolute merger rate value at given redshifts. Lotz et al. (2010) suggest that differences in the range of mass ratios measured by different techniques and differing parent galaxy selection also likely contribute to the variation between studies.

In this paper, our aim is to better constrain the number density of dry mergers at early merging stages and to test the hot halo quenching model. Our analysis is based on imaging data from the Deep component of the Canada-France-Hawaii Telescope Legacy Survey (CFHTLS-Deep) and spectroscopic follow-up with the $6.5 \mathrm{~m}$ Magellan and $5.1 \mathrm{~m}$ Palomar Telescopes. Combining these data sets allowed us to define a sample of close kinematic pairs with redshifts $z=0.1-1.2$. We investigate (1) the number of red-red, blue-red, and blue-blue galaxy pairs as a function of stellar mass, and (2) the relationship between the visible-wavelength colors of close pairs and the galactic halo masses in which they reside. In a companion paper, Chou et al. (2011), we constrained the dry merger number density at late merging stages, and in this paper we seek to undertake a complementary analysis of systems in the early stages of merging. By comparing the number density of dry mergers at different merging stages, we hope to clarify whether star formation is enhanced or quenched (or both, at different epochs) by mergers.

The paper is organized as follows. Section 2 gives the details of the sample selection, data reduction, and the derivation of galaxy properties (stellar masses, color, spectroscopic redshifts, etc.). Results are presented in Section 3, followed by the discussion in Section 4. Our conclusions are presented in Section 5. Throughout this paper, we adopt a concordance cosmology with $H_{0}=70 \mathrm{~km} \mathrm{~s}^{-1} \mathrm{Mpc}^{-1}, \Omega_{M}=0.3$, and $\Omega_{\Lambda}=0.7$.

\section{OBSERVATIONS}

\subsection{Photometry and Galaxy Properties}

The optical photometry used in this paper primarily comes from two of the (CFHTLS) deep survey fields. Together, these fields (denoted as D1 and D4) cover an area of $2 \mathrm{deg}^{2}$. Another small fraction $(\sim 0.6 \%)$ of the sample comes from the D2 field. The CFHTLS deep survey has high-quality broadband photometry in five bands $\left(u^{*}, g^{\prime}, r^{\prime}, i^{\prime}\right.$, and $\left.z^{\prime}\right)$ and the depth of the survey ranges from $26.0\left(z^{\prime}\right)$ to $27.8\left(g^{\prime}\right)$. The typical seeing for the final stacks is 0 '.7-0.'8 in the $i^{\prime}$ band. We utilized the imaging stacks from the Supernovae Legacy Survey (SNLS; Sullivan et al. 2006) for all of our objects. The source extraction and photometry were performed on each field using SExtractor (Bertin \& Arnouts 1996) in dual image mode. The source detection was performed in the $i^{\prime}$ filter $\left(i^{\prime} \sim 26.3\right)$, after applying a bad pixel mask, to avoid noisy or contaminated regions caused by spikes or halos of bright stars. The total area masked is less than $10 \%$ for each field.

Galaxy properties such as stellar mass and star formation rate were derived by comparing the five broadband photometry to a set of template spectral energy distributions (SEDs). The best-fit SEDs were determined through a standard minimum $\chi^{2}$ fitting between the template SEDs and the observed fluxes. The template SEDs were computed by the PEGASE-II galaxy evolution code (Fioc \& Rocca-Volmerange 1997; Le Borgne \& Rocca-Volmerange 2002; Le Borgne et al. 2004) and were convolved with the CFHT filters. A more detailed description 
of the SED fitting analysis that was performed on this data set using the Z-Peg code (Bolzonella et al. 2000; Le Borgne \& Rocca-Volmerange 2002) can be found in Bridge et al. (2010) and Chou et al. (2011). The stellar mass for each pair member was estimated using the Z-Peg code by integrating the total star formation history (SFH) of the best-fit model up to the best-fit age and subtracting off mass loss from late stages of stellar evolution. The galaxy properties of close pairs were taken from the SNLS catalog. An upper limit of photometric redshift $z<1.2$ was applied for the sample presented in this paper because (1) there are a limited number of galaxies with robust spectroscopic redshifts $z>1.2$ to calibrate the photometric redshifts and (2) because the difference between the spectroscopic and photometric redshift becomes too large to derive accurate galaxy properties.

Although the galaxy properties of most close-pair galaxies are derived based on five broadband photometries and the best-fit photometric redshift, we ran the Z-Peg code again for close-pair galaxies with confident spectroscopic redshift measurements (see Section 2.4 for the measurement of spectroscopic redshifts) by replacing the best-fit photometric redshifts with confident spectroscopic ones to obtain precise galaxy properties. This indicates that the results presented in Figures 4 and 5 are based on confident spectroscopic redshift measurements.

\subsection{Spectroscopy of the Photometric Sample}

This sample described in the previous section was used to define targets for a three-night spectroscopic program undertaken using the Inamori-Magellan Areal Camera and Spectrograph (IMACS) on the Magellan Baade $6.5 \mathrm{~m}$ telescope in 2005 October and 2006 September. SCOPIC, a Spectroscopic Study of Close Optical Pairs In CFHTLS (PI Bridge), was aimed at identifying a large sample $(\sim 1500)$ of kinematic close galaxy pairs in order to further understand the galaxy merger rate and the connection between mergers, star formation, and AGN activity. Close-pair candidates were selected using the following criteria: (1) a projected separation between pair members less than $50 h^{-1} \mathrm{kpc}$; (2) an apparent $i^{\prime}$ magnitude difference $\leqslant 1.5 \mathrm{mag}$, in order to select roughly equal mass or major mergers; and (3) a photometric redshift difference between pair members of $\Delta z<0.15(1+z)$. This selection resulted in a sample of 2730 galaxies that resided in a close pair (or triple system) of galaxies over $2 \mathrm{deg}^{2}$ and were spectroscopically targeted.

Figure 2 shows the distribution of projected separations for the potential close pairs in the fields chosen, along with our sampling of this distribution. The black solid curve represents the projected separation distribution for all of the potential close pairs presented in D1 and D4 fields that satisfy the above-mentioned selection criteria. The green solid and dashed curves represent the projected separation distribution of total and non-detected close pairs, respectively. The red dashed curve represents the projected separation distribution for detected close pairs (both pair members have confident redshift measurements; see Section 2.4 for more details). To increase the statistics, both green and red curves have a bin size of $5 h^{-1} \mathrm{kpc}$. There is clearly a significant drop in our sampling of the parent population of potential close pairs when the projected separation is greater than $20 h^{-1} \mathrm{kpc}$, which indicates a selection bias for close pairs with smaller projected separations. This selection bias is due to a higher selection priority of potential close pairs with projected separations smaller than $20 h^{-1} \mathrm{kpc}$. Therefore, all the results presented in the present paper focus mainly on close pairs with projected separations smaller than $20 h^{-1} \mathrm{kpc}$.
Slit masks were designed to maximize the opportunity to get redshifts for both members of a close pair, by placing either a separate slit on each member or using a single slit that was tilted to cover both galaxies. IMACS has one short $(f / 2)$ and one long $(f / 4)$ focal ratio camera. The observations were conducted with the short camera and the $8 \mathrm{~K} \times 8 \mathrm{~K}$ Mosaic2 CCD detector, providing a field of view of 27.4 in diameter. We used the grating with a groove density of 200 lines $\mathrm{mm}^{-1}$ and the diffraction order $m$ of 1 to achieve the spectral coverage of 5000-9000 А with the central wavelength at $6600 \AA$. The slit width was set to $1^{\prime \prime}$ and the wavelength dispersion was $\sim 10 \AA$ per slit, or equivalent to a resolving power of 660 . The individual exposure times were 30 minutes with a total average integration time for each slit mask of $1.5 \mathrm{hr}$. The data reduction was performed using the Carnegie Observatories System for MultiObject Spectroscopy (COSMOS) software package, developed by A. Oemler, K. Clardy, D. Kelson, and G. Walth, which is publicly available at code.obs.carnegiescience.edu/cosmos. We used COSMOS to align slit masks, correct flat fields, subtract sky background, and perform flux calibrations for IMACS data. The flux calibration stars were LTT1788 and gd71 for the D1 and D4 fields, respectively. The SED of the standard stars was taken from Hamuy et al. (1992, 1994) and Bohlin et al. (1995). A $3 \sigma$ rejection threshold was used to remove cosmic rays when stacking subframes from the same slit mask. One-dimensional spectra were extracted using an IDL program we wrote for this purpose.

Although the vast majority of the data described in this paper comes from the Magellan Baade telescope via the SCOPIC survey, as a test of our methodology we also obtained spectra for a small sample of 20 (mainly red) close pairs in the CFHTLS D2 using the Palomar 200 inch telescope. The purpose of investigating this small sample was to verify that red galaxies are detectable using our techniques; we will occasionally refer to these Palomar observations when describing the effectiveness of our methodology of this paper, but to preserve sample homogeneity we do not include any Palomar data in our figures or analysis. These pairs were observed using the Double Spectrograph (DBSP) on the Palomar 200 inch telescope in 2010 March. DBSP has a blue and a red spectrograph that work simultaneously to provide a spectral coverage of 3000-7000 $\AA$ for the blue camera and 4700-10000 $\AA$ for the red camera. The slit length of the DBSP long-slit mode is $128^{\prime \prime} .0$ and the slit width varied from $1^{\prime \prime} .0$ to $1^{\prime \prime} .5$ depending on weather conditions in order to achieve the highest signal-to-noise ratio. We used a grating with a groove density of 1200 lines $\mathrm{mm}^{-1}$ that yields a wavelength dispersion of $1.38 \AA$ and $2.1 \AA$ for $1^{\prime \prime} .0$ and $1^{\prime \prime} .5$ slits, respectively, at the diffraction of the order of $m=1$. The blazed wavelength of the red and blue spectrographs was set to $7100 \AA$ and $4700 \AA$. The individual exposure time was 10 minutes, with a total integration time of $1 \mathrm{hr}$ per galaxy. The data reduction was done using a combination of standard IRAF routines and our own IDL code for extraction.

\subsection{Color Classification}

Galaxies were classified as red or blue based on the stellar mass-dependent color-selection criteria introduced in Chou et al. (2011). In this process, galaxies are subdivided on the color-mass plane according to position relative to the following line:

$$
\left(g^{\prime}-i^{\prime}\right)_{\text {rest }}=-0.0076+0.13 \times M_{\text {stellar }}-0.1 \text {. }
$$




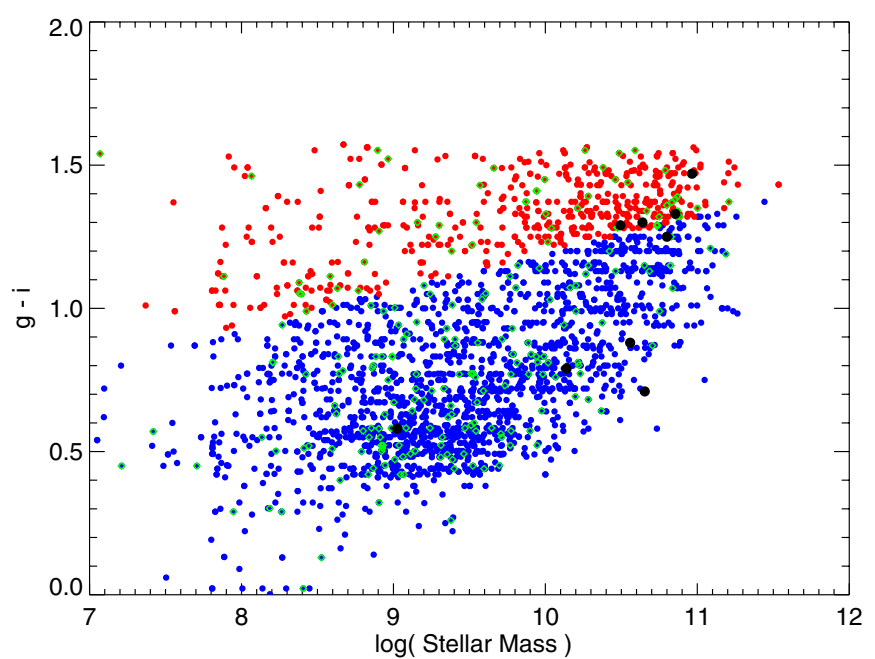

Figure 1. Rest-frame $g-i$ color vs. stellar mass for close-pair galaxies in the D1, D2, and D4 fields. Red and blue dots represent the red and blue galaxies classified via the fiducial color as shown in Equation (1). Black dots are D1 galaxies with MIPS $24 \mu \mathrm{m}$ detection. Green diamonds represent close pairs in which both galaxies are detected. Note that red pairs also span a wide range in stellar mass, showing that there is no selection bias toward the bright red pairs. We artificially assign objects with $24 \mu \mathrm{m}$ detection as blue galaxies and excluded objects with photometric redshifts greater than 1.2. The sample ends up with 607 red and 2143 blue galaxies.

(A color version of this figure is available in the online journal.)

The rest-frame $g^{\prime}-i^{\prime}$ color is obtained from the Z-Peg fitting results. If the rest frame $g^{\prime}-i^{\prime}$ color of a galaxy is greater than the fiducial color defined by the line, then the galaxy is classified as red, and vice versa for blue galaxies. Figure 1 shows the restframe $g^{\prime}-i^{\prime}$ color versus the stellar mass of close-pair galaxies in the D1, D2, and D4 fields. Red and blue dots represent red and blue galaxies, respectively. The black dots represent galaxies with the Spitzer Multiband Imaging Photometer for SIRTF (MIPS) $24 \mu \mathrm{m}$ detection (down to a flux limit of 340 $\mu \mathrm{Jy})$. Cowie \& Barger (2008) report that at $z<1.5$, most red galaxies with a $24 \mu \mathrm{m}$ flux $>80 \mu \mathrm{Jy}$ fall into the blue cloud after the appropriate dust extinction corrections are applied. To account for the color change in dusty sources, we artificially assign the black dots with the rest-frame $g^{\prime}-i^{\prime}$ color greater than the fiducial color to the blue cloud. Note that MIPS $24 \mu \mathrm{m}$ observations do not include the D4 field, so the black dots in Figure 1 correspond to galaxies from the D1 field only. However, there are only 10 objects in the D1 field with a MIPS $24 \mu \mathrm{m}$ detection, which is less than $1 \%$ of the sample in the D1 field. It is reasonable to assume that the number of galaxies with MIPS $24 \mu \mathrm{m}$ detection in the D4 field is similarly low, and therefore this should not affect the overall result. After the correction for $24 \mu \mathrm{m}$ detections, the sample is comprised of 607 red galaxies and 2143 blue galaxies. Green diamonds in Figure 1 represent close pairs where both members have spectroscopic redshift measurements.

\subsection{Spectroscopic Redshifts}

Redshifts were obtained from our spectra based on emission line identifications and cross-correlation against blue and red galaxy templates. The emission line identification method is made straightforward by recognizing multiple emission lines using their relative positions on the wavelength axis. The redshift is determined by fitting a Gaussian function to the identified brightest emission line. The corresponding redshift and the $1 \sigma$ error can be derived from the central wavelength and the $1 \sigma$

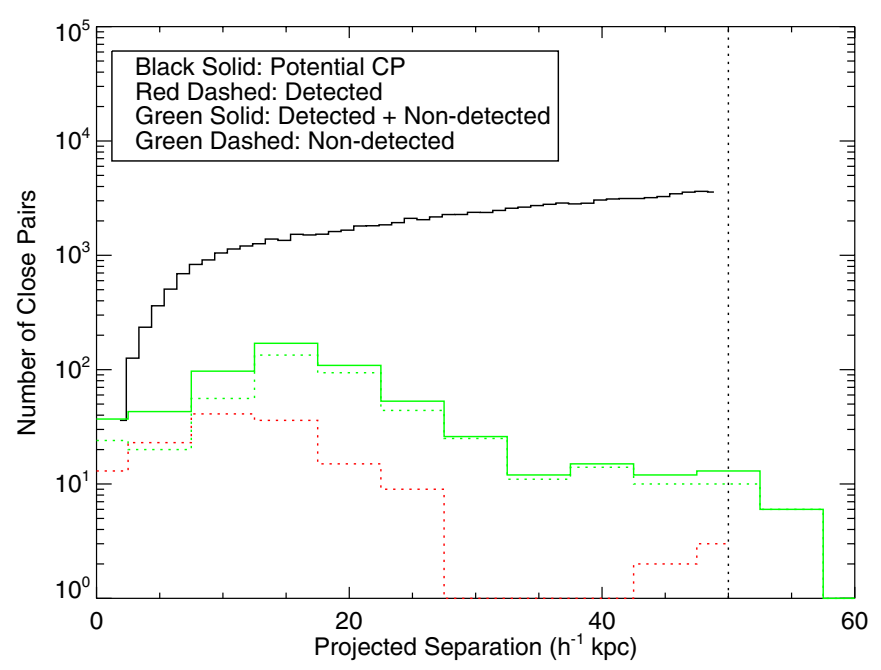

Figure 2. Analysis of the selection biases in our sample as a function of projected separation. The black curve represents the projected separation distribution of all the potential close pairs presented in the D1 and D4 fields which satisfy the close-pair selection criteria. The green solid and dashed curves represent the projected separation distribution of total and non-detected close pairs presented in this work; the red dashed curve represents the projected separation distribution of detected close pairs (i.e., both pair members have confident redshift measurements; see Section 2.4 for more details). Note that there is a significant drop when the projected separation is greater than $20 h^{-1} \mathrm{kpc}$ in the green and red curves, which indicates a selection bias for close pairs with smaller projected separations.

(A color version of this figure is available in the online journal.)

error of the fitting function. For the majority of the continuum detected galaxies, emission lines are obvious and relatively easy to identify; however, in a small number $(\sim 20 \%)$ of cases, emission lines are hard to identify or only the continuum can be seen (e.g., very red elliptical galaxies). For those galaxies, we used the cross-correlation fitting method to determine the spectroscopic redshift by identifying absorption features. We developed an IDL program based on the package "C_CORRELATE" and adopted the photometric redshift as a prior guess. To save computation time, we only considered the redshift range of $z_{\text {pri }} \pm 1.0$ (if $z_{\text {pri }}<1$, then we start from $z=0$ ). A blue and a red galaxy template generated from the stellar population synthesis code BC03 (Bruzual \& Charlot 2003) were used during cross-correlation fittings. The templates were constructed using a model that convolves a single stellar population with solar metallicity and an exponentially declining SFH with an $e$-folding timescale $\tau=1 \mathrm{Gyr}$. The star formation cutoff time $t_{\text {cut }}$ was set to $20 \mathrm{Gyr}$. We also applied the dust attenuation to templates using the default values in $\mathrm{BC} 03$ which stated that the total effective $V$-band optical depth $\tau_{V}$ equals 1.0 and the fraction $\mu$ of the contribution from the ambient diffuse ISM equals 0.3 , respectively. Finally, we took the SED with an age of $1.0 \mathrm{Gyr}$ as the blue galaxy template, and the SED with an age of 5.0 Gyr as the red galaxy template. Since the output SEDs from the BC03 do not include the emission lines, we added emission lines artificially to the blue template based on the emission line list used in the DEEP2 survey kindly provided by R. Yan (2011, private communication). The emission line widths in the DEEP2 line list were convolved to the IMACS spectral resolution before being added to the blue template.

We adopted the confidence level classification system introduced by Abraham et al. (2004) for the reliability of the spectroscopic redshifts (see Table 1 for definitions of the confidence levels). Class 1 corresponds to an unreliable redshift, while 
Table 1

Redshift Confidence Classes

\begin{tabular}{|c|c|c|c|}
\hline Class & Confidence & Number of Galaxies & Note \\
\hline \multicolumn{4}{|c|}{ Failures } \\
\hline 0 & None & 1367 & No detection. \\
\hline 1 & $<50 \%$ & 270 & $\begin{array}{l}\text { Only one emission/feature is detected. } \\
\text { The emission line cannot be identified even } \\
\text { with the aid of photometric redshift. }\end{array}$ \\
\hline \multicolumn{4}{|c|}{ Redshifts inferred from multiple features } \\
\hline 2 & $>75 \%$ & 321 & $\begin{array}{l}\text { Reasonably secure. Redshift determined } \\
\text { from two or more emission lines or features. }\end{array}$ \\
\hline 3 & $>95 \%$ & 327 & $\begin{array}{l}\text { Secure. Redshift determined from multiple } \\
\text { emission lines/features }+ \text { supporting continuum. }\end{array}$ \\
\hline 4 & Certain & 168 & Unquestionably correct. \\
\hline \multicolumn{4}{|c|}{ Single line redshifts } \\
\hline 9 & & 297 & $\begin{array}{l}\text { Obvious single line emission. } \\
\text { The inferred spectroscopic redshift is consistent } \\
\text { with the photometric redshift. }\end{array}$ \\
\hline
\end{tabular}

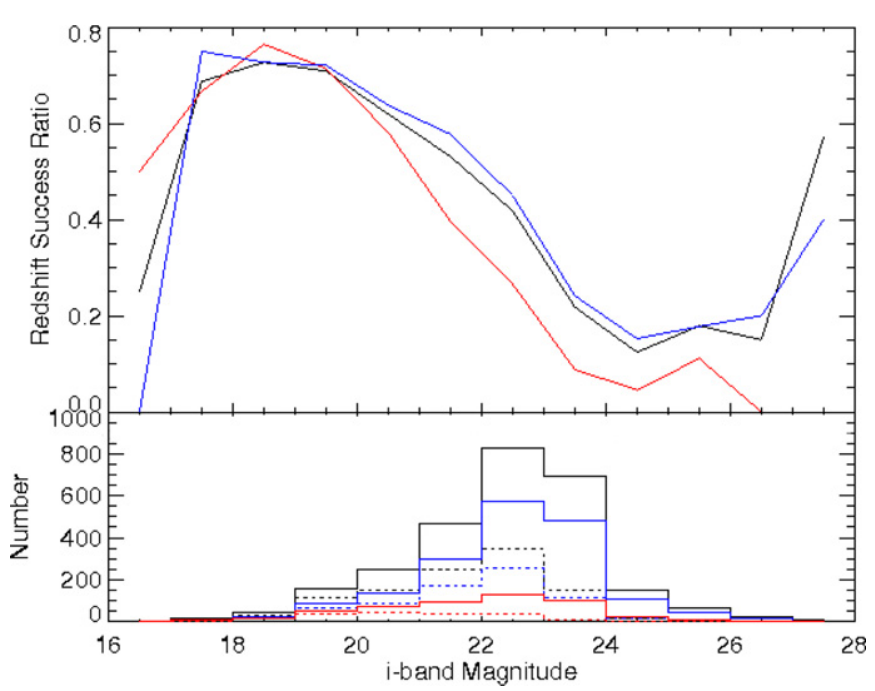

Figure 3. Upper panel: successful redshift ratio vs. $i^{\prime}$-band apparent magnitude. The black solid line shows the successful ratio for total galaxies; the blue and red solid lines show the successful ratio of blue and red galaxies, respectively. Bottom panel: histograms corresponding to the total, red, and blue galaxies shown in black, red, and blue, respectively. Solid histograms represent the total number of galaxies in different magnitude bins, while dashed histograms represent the number of galaxies with a confidence level greater than 1.

(A color version of this figure is available in the online journal.)

class 4 corresponds to a high, reliable redshift. Single emission line objects require special consideration because in most cases a line is detected with certainty, but the identification of the line is uncertain. In such cases, we used photometric redshifts as an auxiliary source of information, and if the inferred spectroscopic redshift is within $1 \sigma$ error of the photometric redshift, we classify the object as having a high-confidence redshift (class 9), otherwise we assign it a low-confidence level (class 1).

Of 2750 galaxies targeted with slits, roughly half of them (1385) have redshift measurements. About 80\% (1115) of these redshifts have confidence levels greater than 1 , which we refer to as successfully detected ones. As expected, the redshift success rate is directly related to the brightness of the galaxy, and Figure 3 shows our magnitude selection function (the fraction of successful redshift measurements as a function of $i^{\prime}$-band magnitude) for the total sample and for red and blue subsets. For galaxies with an $i^{\prime}$-band magnitude brighter than 20 , the success rate for blue galaxies is more or less equal to that of red galaxies, while at fainter magnitudes $\left(i^{\prime}>20 \mathrm{mag}\right)$ the rate is on average $\sim 20 \%$ higher for blue galaxies. This is understandable, as blue galaxies, having active star formation, often exhibit emission lines that are easily detected even when continuum measurements needed for absorption-line redshifts are impossible to obtain. The lower panel of Figure 3 shows the absolute number of galaxies in our sample as a series of histograms. The solid lines represent the total number of galaxies, while the dashed lines represent the total number of galaxies with confidence levels greater than unity. The histograms show a fairly broad peak spanning $22<i^{\prime}<$ $24 \mathrm{mag}$, and at this point the selection function corresponds to $\sim 40 \%$ successful detections. ${ }^{3}$

In summary, we have obtained redshifts for 1385 galaxies in the range $0.1<z<1.2$, and 1115 of these $(\sim 80 \%)$ have high-confidence measurements. Table 1 shows the number of galaxies in each confidence level.

\section{RESULTS}

Figure 4 shows the relative velocity versus physical separation for all close pairs in our sample in which both members have high-confidence redshifts. The format of this figure resembles that of Figure 5 in Patton et al. (2000), but with a number of key differences, which we will now proceed to describe.

The central difference between our analysis and that of Patton et al. (2000) is that we have attempted to subdivide pairs by the stellar populations of their member galaxies. We distinguish between "red-red" pairs (systems in which both galaxies are quiescent), "blue-red" pairs (systems in which one galaxy is quiescent while the other is star forming), and "blue-blue" pairs (systems in which both galaxies are star forming). Red, green, and blue colors in Figure 4 represent red-red, blue-red, and blue-blue pairs, respectively. Since none of the galaxies

\footnotetext{
3 The success rate is similar for the sample of 20 galaxies used to test our methodology with the Palomar 200 inch. Roughly half of the Palomar galaxies were not detected due to bad weather conditions, although nine pairs were observed in good weather conditions. We obtained six spectroscopically confirmed close pairs (12 galaxies) out of nine pairs (18 galaxies), which corresponds to a redshift success rate of $\simeq 70 \%$. Among the six spectroscopically confirmed close pairs, one was a blue-blue pair and the remainder were red-red pairs. The members of these pairs have $i^{\prime}$-band magnitudes brighter than 20 .
} 


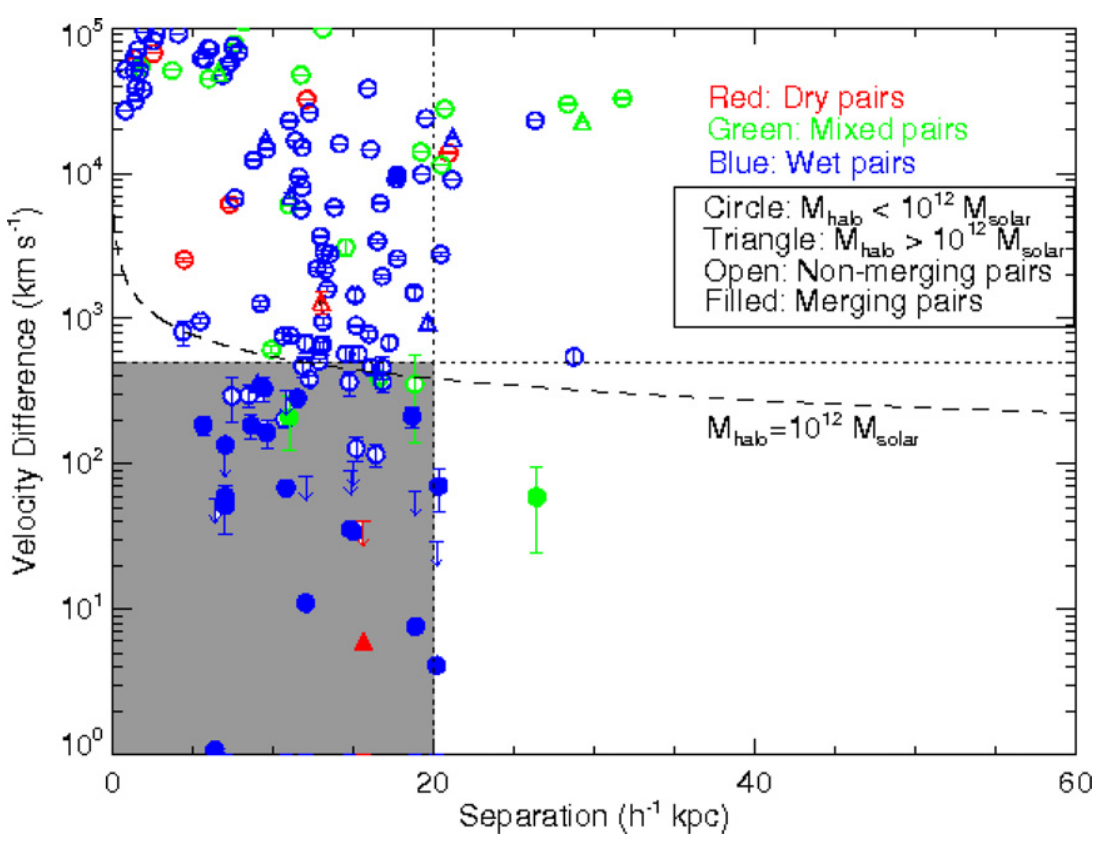

Figure 4. Diagram of separation vs. velocity difference for close-pair galaxies. Red, green, and blue colors represent red-red, blue-red, and blue-blue pairs, respectively. Circle and triangle symbols represent galaxies with their total halo masses smaller and greater than $10^{12} M_{\odot}$ (equivalent to a stellar mass of $10^{11} M_{\odot}$ using the stellar to total halo mass ratio of 0.1 taken from Gonzalez et al. 2007). Filled symbols represent pairs with their separation smaller than the corresponding virial radii, which are classified as merging pairs. Two dotted lines indicate a separation of $20 h^{-1} \mathrm{kpc}$ and a velocity difference of $500 \mathrm{~km} \mathrm{~s}^{-1}$. The bottom left gray corner defined by the two dotted lines shows the conventional real merging pair selection area suggested by Patton et al. (2000). The dashed lines represent the virial radius as a function of velocity difference of a system with a halo mass of $10^{12} M_{\odot}$. Before the selection of merging close pairs, there are 148 spectroscopic pair candidates $(8$ red-red pairs, 29 blue-red pairs, and 111 blue-blue pairs on the plot). Note that none of the close pairs presented on the diagram has MIPS24 $\mu \mathrm{m}$ detection (flux limit equals to $340 \mu \mathrm{Jy}$ ). After the pair selection based on the virial radius assumption using the stellar to total halo mass ratio equal to $0.1,21$ out of 148 pairs are merging pairs. The 21 selected pairs are composed of 1 red-red pair, 2 blue-red pairs, and 18 blue-blue pairs.

(A color version of this figure is available in the online journal.)

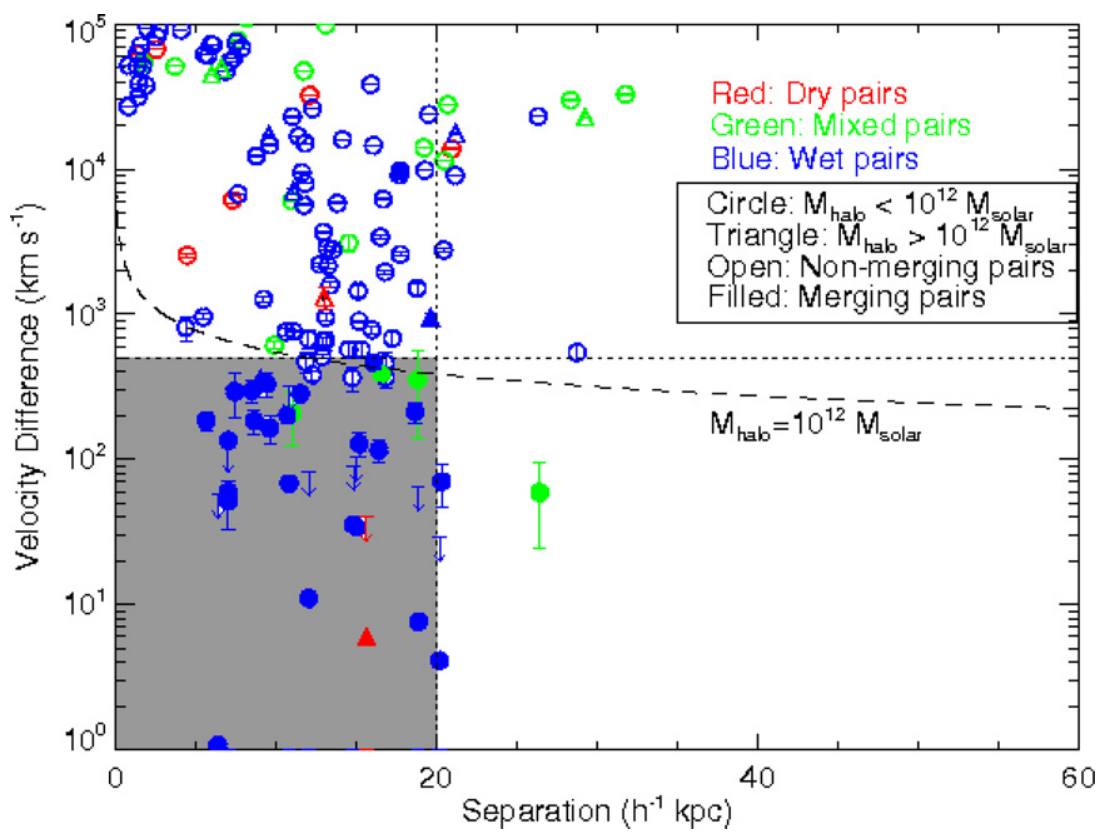

Figure 5. Diagram of separation vs. velocity difference for close-pair galaxies using different stellar to total halo mass ratios taken from Leauthaud et al. (2011). This figure is presented in the same way as Figure 4. It is clear that the number of filled symbols increases, and most of them are still located within the conventional merging pair selection area. After the application of different stellar to halo mass ratios, 30 out of 148 are classified as merging pairs. Of these, 1 is a red-red pair, 4 of them are blue-red pairs, and 25 of them are blue-blue pairs. Table 2 summarizes the number of pairs selected by different criteria.

(A color version of this figure is available in the online journal.)

presented in Figures 4 and 5 have Spitzer MIPS $24 \mu \mathrm{m}$ detection, contamination of the quiescent red galaxy sample by very dusty star-forming systems is probably negligible. There are 148 pairs (exclude the biased selected D2 samples) selected on the basis of photometric redshift, where both members have confident spectroscopic redshift measurements, and we will show in a moment that only around one-fifth of these turn out to be physical pairs once spectroscopic redshift and velocity 
information is incorporated. Among the above-mentioned 148 candidate pairs, 8 are red-red pairs, 29 are blue-red pairs, and 111 are blue-blue pairs.

In addition to segregating pairs by their dominant stellar populations, Figure 4 also subdivides pairs into two bins on the basis of their estimated total (i.e., dynamical) masses. We adopt the simplistic approach of estimating the system's total mass by simply scaling each galaxy's stellar mass by a constant total mass-to-stellar mass ratio of 10:1 taken by Gonzalez et al. (2007) and then summing the masses (we will describe a somewhat more refined approach later in this paper). Subdivision into high-mass and low-mass bins occurs at a total mass of $10^{12} M_{\odot}$ because, as noted earlier, this is the mass threshold predicted by the hot halo model. Circles and triangles in Figures 4 and 5 represent pairs with total halo masses less than and greater than $10^{12} M_{\odot}$.

Patton et al. (2000) note that close pairs with separations of less than $20 \mathrm{~h}^{-1} \mathrm{kpc}$ and velocity differences of less than $500 \mathrm{~km} \mathrm{~s}^{-1}$ generally show clear morphological signs of galaxy-galaxy interactions. Our own visual inspection, described earlier, concurs with Patton et al.'s impression and we therefore adopt these criteria as a useful starting point for our own more detailed analysis. Patton et al.'s limits are shown as two dotted lines in Figure 4, and in the present paper we will treat pairs inside these limits (the light gray region at the lower left corner of the figure) as probable merging pairs. Figure 4 shows a gap at separations smaller than $5 h^{-1} \mathrm{kpc}$ and velocity differences $\Delta v<10,000 \mathrm{~km} \mathrm{~s}^{-1}$. This gap originates from an inevitable ambiguity that arises when distinguishing between close pairs and on-going mergers at very small separations. The former were targeted by our investigation, but the latter were not. To verify our explanation for the gap in the figures, we inspected both the two-dimensional spectra and the $i^{\prime}$-band images of all galaxies seen at small separations, and compared them with merging systems from the sample in Bridge et al. (2010). Objects located at the upper left corner $(\Delta v>10,000 \mathrm{~km}$ $\mathrm{s}^{-1}$ ) of the figures correspond to physically unconnected pairs seen at small separations because of projection effects. While these objects were generally both placed on a single slit, they exhibited no obvious tidal features on the $i^{\prime}$-band images, so that the catalog construction process and subsequent morphological classification treated them as independent galaxies.

In addition to the gap at small separations, Figure 4 shows a paucity of close pairs with separations greater than $20 h^{-1} \mathrm{kpc}$. This originates from the selection bias against systems with large separations described in Section 2.2.

If we adopt Patton et al.'s methodology, then there are 31 probable merging pairs isolated in Figure 4. It is interesting to note that 27 of these are blue-blue pairs, 3 are blue-red pairs and 1 of them is a red-red pair. This strongly suggests that red-red mergers are quite rare at $z \sim 0.5$ (at least for close pairs with their separation smaller than $20 h^{-1} \mathrm{kpc}$ ), which is consistent with the reported decline in "dry" merging activity at such redshifts reported in Chou et al. (2011). To our magnitude limit, blue galaxies outnumber red galaxies by nearly a factor of four: the parent sample contains 607 red galaxies ( $\sim 22 \%$ of the total sample) and 2143 blue galaxies ( $\sim 78 \%$ of the total sample). The most conservative assumption is to assume that merging has no impact whatsoever on the colors of galaxies and that galaxies of different color are spatially uncorrelated, in which case the colors of close pairs can simply be modeled as a binomial distribution with a $22 \%$ probability that a galaxy is red. In this case, we expect the fraction of red-red pairs to be
$(0.22)^{2}$, corresponding to a $4.8 \%$ red fraction, and therefore we expect to see 1.5 red-red pairs among our sample of 31 physical pairs. This is consistent with the single detection of a red-red merging pair.

However, a legitimate criticism of the analysis presented above is that our definition for what constitutes a probable merging pair is non-unique. For example, Lin et al. (2004a, 2008) advocate a maximum separation of $50 h^{-1} \mathrm{kpc}$ rather than $20 h^{-1} \mathrm{kpc}$. If we extend our sample out to this limit, then there would be one more blue-red and two more blue-blue merging pairs. ${ }^{4}$ Given the somewhat arbitrary nature of the limits chosen, it is worthwhile considering whether or not a more physically motivated definition for what constitutes a merging pair might not be preferable. Therefore, inspired by the idea of a halo occupation number (Lin et al. 2004b; Hopkins et al. 2010), we propose the following simple definition: a merging pair is a system of two galaxies whose projected separation $r_{p}$ is equal to or less than two times the total system's virial radius. In other words, the pair can be thought of as sharing a common halo if $r_{p}<2 r_{\text {virial }}$, where $r_{\text {virial }}=G M / V^{2}$, with $G$ representing the gravitational constant, $M$ representing the total halo mass of the system, and $V$ representing the threedimensional relative velocity of the two pair members. Since the actual three-dimensional relative velocity is not available, we must multiply line-of-sight velocity differences by a factor of $\sqrt{3}$ as a statistical correction when computing $r_{\text {virial }}$ from redshifts. 5

For reference, the virial radius of a system with a total mass of $10^{12} M_{\odot}$ and a velocity difference of $500 \mathrm{~km} \mathrm{~s}^{-1}$ is $12 h^{-1} \mathrm{kpc}$. The dashed curve in the figure maps the variation of virial radius as a function of velocity difference for a system with a halo mass of $10^{12} M_{\odot}$. In our approach, any system with a total mass of $10^{12} M_{\odot}$ would be considered a merging pair if it lies anywhere below this curve. We applied this methodology to systems of all masses by assuming a certain total mass-to-stellar mass ratio, and in Figure 4 we flag merging pairs determined in this manner using filled symbols. Using this approach, 21 systems (around $65 \%$ of the pairs in the boxed region at the lower left corner of Figure 4) are found to be merging. One of them is a red-red system and two pairs are blue-red systems, but 18/21 (86\%) of the pairs are blue-blue systems.

A criticism that can be directed at Figure 4 is its heavy reliance on a uniform stellar-to-total mass ratio in order to infer virial radii. As described earlier, the total masses (and hence virial radii) used to construct Figure 4 have been computed using the rather simplistic approach of assigning a total mass to a composite system by assuming a constant total mass-to-stellar mass ratio of 10:1. This commonly adopted (Conroy et al. 2007; Spitler et al. 2008) total mass-to-stellar mass ratio is taken from Gonzalez et al. (2007), who derived it from galaxy groups and clusters with total halo masses $\geqslant 10^{14} M_{\odot}$, which are about two orders of magnitude more massive than the total masses of the systems in our sample. Naturally, other measurements

\footnotetext{
4 It is worth recalling that the Milky Way is about $750 \mathrm{kpc}$ from M31, with a velocity difference of about $120 \mathrm{~km} \mathrm{~s}^{-1}$ (Karachentsev et al. 2004; de Vaucouleurs et al. 1991). Even though our ultimate destiny seems to be to merge with the Andromeda galaxy, the present configuration of the Local Group would not result in the Milky Way + M31 system being flagged as a merging pair by any of the criteria adopted here.

5 The factor of $\sqrt{3}$ is obtained by assuming an isotropic velocity difference distribution, so that the relationship between the pairwise velocity $V$ and its components $V_{x}, V_{y}$, and $V_{z}$ can be simply expressed as $\sqrt{3}$ times the line-of-sight velocity: $V=\sqrt{V_{x}^{2}+V_{y}^{2}+V_{z}^{2}}=\sqrt{3 V_{z}^{2}}=\sqrt{3} V_{z}$.
} 
Table 2

Summary of Three Types of Close Pairs Selected Using Different Criteria

\begin{tabular}{|c|c|c|c|}
\hline Criteria & $\begin{aligned} r_{p} & <20 h^{-1} \mathrm{kpc} \\
\Delta v & <500 \mathrm{~km} \mathrm{~s}^{-1}\end{aligned}$ & $\begin{array}{c}r_{p}<2 r_{\text {virial }} \\
(\text { Gonzalez et al. 2007) }\end{array}$ & $\begin{array}{c}r_{p}<2 r_{\text {virial }} \\
\text { (Leauthaud et al. 2011) }\end{array}$ \\
\hline Red-red pairs & 1 & 1 & 1 \\
\hline Blue-red pairs & 3 & 2 & 4 \\
\hline Blue-blue pairs & 27 & 18 & 25 \\
\hline Total & 31 & 21 & 30 \\
\hline
\end{tabular}

of the total mass-to-stellar mass ratio can be found in the literature (for example, Conroy et al. 2007; Giodini et al. 2009; Leauthaud et al. 2010) but most such work focuses either on limited classes of objects (e.g., early-type galaxies; Heymans et al. 2006) or on composite systems with masses higher than $10^{13} M_{\odot}$ (Mandelbaum et al. 2006).

Fortunately, statistical analyses of stellar-to-total mass ratios based on weak lensing are now beginning to emerge, and these can better inform our analysis by allowing us to incorporate a total-to-stellar mass ratio that is a function of stellar mass. Table 2 summarizes the number of pairs selected by adopting a variable total-to-stellar mass ratio using the weak-lensingbased results presented by Leauthaud et al. (2011) and provides a comparison with the other methods we have described. We emphasize that weak-lensing measurements are constructed from large samples in the appropriate mass range for our purposes, but at present such investigations are independent of galaxy morphology. The results presented by Leauthaud et al. (2011) are based on COSMOS imaging data (Scoville et al. 2007) coupled to follow-up spectroscopic redshifts from the zCOSMOS survey (Lilly et al. 2007). In Figure 5, we incorporate these data in an attempt to improve upon Figure 4. The format of Figure 5 is identical to that of Figure 4, except that nonconstant stellar-to-total mass ratios have been used to infer the virial radii. As a result, there are 31 physical pairs out of 148 pairs shown in Figure 5, and we again flag merging pairs using filled symbols. Leauthaud et al. (2011) probe systems with halo masses down to below $10^{11} M_{\odot}$, making this an excellent fit to our sample. However, it should be emphasized that weaklensing analyses are fairly new and subject to a large number of subtle systematics, so some caution should be used in the interpretation of Figure 5. We suspect that Figure 5 is likely to have more long-term value than Figure 4, but the latter provides a robust backstop against which Figure 5 can be compared.

\section{DISCUSSION}

\subsection{Abundance of Physical Pairs}

A comparison of Figures 4 and 5 makes it clear that the use of a non-constant mass-to-light ratio based on weak-lensing data results in an increase in the number of physical pairs. This is because the typical total-to-stellar mass ratios for galaxyscale halos are much higher than the corresponding value for groups or clusters, so the derived virial radii are also larger. In Figure 5, 1 physical pair is a red-red system, 4 physical pairs are blue-red systems, and 25 physical pairs are blue-blue systems. Although red-red pairs are clearly rare, their absence is not due to our experimental design: when we augmented the IMACS data with Palomar observations purposely designed to find red-red systems with our technique, we did find them: the Palomar observations resulted in five red-red pairs and one blue-blue pair, and the redshift success rate is consistent with that of IMACS observations. ${ }^{6}$

Overall, 30 out of 148 spectroscopic pair candidate pairs presented in Figure 5 turn out to be physical pairs. While the majority of these occupy the light gray region in the diagram that Patton et al. (2000) suggest corresponds to systems with clear indications of tidal disturbance, around $10 \%$ of the physical mergers lie outside this region. These are typically pairs with one rather massive galaxy (resulting in a total system halo mass greater than $10^{12} M_{\odot}$, denoted by the triangle symbols in Figure 5), although two are pairs with relatively low-velocity differences but larger separations. The latter galaxies would likely be missed by the traditional close-pair selection methods for identifying physical merging pairs, since it would be rather risky to select merging pairs by simply selecting on the basis of higher velocity differences or larger separations, which would result in many false detections. It is also interesting to note that we find a number of systems $(\sim 15 \%)$ that are unlikely to be physical pairs even though they are located in the bottom left corner of Figure 5. These are likely to be "false positives" inferred using the Patton et al. (2000) methodology, and these systems emerge because their halo masses are low, resulting in small virial radii. Of course, our method has false positives of its own: since the velocities used in our analysis are line of sight, and therefore a combination of projected galaxy peculiar velocities and cosmological redshifts, and some fraction of these pairs are likely to have true separations of several Mpc and are unlikely to merge. Such false positives are inevitable in any physical pair selection technique, and the net result is that the number of close physical pairs is likely to be slightly overestimated.

It is worthwhile to consider the ultimate fate of the physical pairs identified in the present survey. We assume that they will all eventually merge and become single systems. However, as noted in the Introduction, the timescale over which this will occur is far from certain, and the question of whether mergers trigger or suppress star formation remains open. We will have more to say about this later in the discussion in the context of the Hot Halo model, but for now it is worthwhile to point out that Figure 5 reveals a rather striking discrepancy in the fraction of red-red "pairs" that are likely to be line-of-sight optical superpositions, relative to the number of physical red-red pairs. This is clearly seen even if one simply uses the straightforward definition for a physical merger suggested by Patton et al. (2000) and compares the fraction of pairs that are red-red systems inside the box $(\sim 1 \%)$ to the corresponding fraction $(5 \%)$ of pairs that are red-red systems in regions of the diagram that are outside the box.

\footnotetext{
6 We again emphasize that Palomar observations are not included in the summary statistics presented in this paper (e.g., in any of the discussion below), since they were intentionally biased and are mainly useful for testing our methodology.
} 


\subsection{Comparison with DEEP2 and VVDS Surveys}

Because the overall completeness of our survey (shown in Figure 2) is relatively low, we have mainly focused on making relative comparisons between the properties of physical pairs and field galaxies, since the photometric and spectroscopic selection criteria for the two populations are the same. However, it is worthwhile to consider the absolute numbers of objects seen, along with the merger rate density of close pairs obtained from other surveys. A comparison with results from a merger analysis of the DEEP2 survey (Lin et al. 2008) is particularly interesting, because these authors also selected close pairs based on dynamical criteria (i.e., separations $<30 h^{-1} \mathrm{kpc}$ and velocity difference $<500 \mathrm{~km} \mathrm{~s}^{-1}$ ). ${ }^{7}$

The present IMACS survey's comoving volume is two square degrees over a redshift range of $0.1<z<1.2$, which corresponds to $1.1 \times 10^{7} \mathrm{Mpc}^{3}$. Using a merger rate density of $\sim 1.0 \times$ $10^{-3} h^{3} \mathrm{Mpc}^{3} \mathrm{Gyr}^{-1}$ (where $h=0.7$ ) and a merging timescale of $0.5 \mathrm{Gyr}$ for all pairs taken from Figure 7 in Lin et al. (2008), one expects there to be $\simeq 1900$ close pairs in our survey volume. Figure 5 shows that our survey contains 31 physical pairs. While this number seems low, in fact it matches our expectations based on the selection criteria presented in Figures 2 and 3. In Figure 3 , near the peak of the galaxy count distribution at around $i \sim 22.5 \mathrm{mag}$, the spectroscopic completeness is around $\sim 40 \%$ for an individual galaxy, and this should be squared to compute the pairwise completeness (as a first-order approximation, to account for the fact that we require successful redshifts for both galaxies in the pair). So the overall spectroscopic completeness of galaxies targeted with slits is around $16 \%$. This must in turn be multiplied by the sparse sampling completeness factor which accounts for the fact that only a subset of galaxies was targeted with a slit. In Figure 2, we see that the ratio of the parent sample to the observed sample is roughly 10:1 for potential pairs with separations smaller than $20 h^{-1} \mathrm{kpc}$. Therefore, our 31 physical pairs map to $31 / 0.16 \times 10=1937$, which is in good agreement with the expected $\simeq 1900$ physical pairs predicted from the merger rate density of the DEEP2 survey.

Our main conclusion (that dry mergers are rare at intermediate redshifts) has been foreshadowed by Lin et al. (2008), using the data from the DEEP2 Survey. These authors report that the red-red pair merger rate density within the redshift range $0.2<z<1.2$ is $\sim 1.0 \times 10^{-4} h^{3} \mathrm{Mpc}^{-3} \mathrm{Gyr}^{-1}$, with a merging timescale of 0.5 Gyr. Using this red-red pair merger rate density and a merging timescale of $0.5 \mathrm{Gyr}$, we can estimate the number of red-red pairs predicted from the DEEP2 survey result given our IMACS survey volume. Again, using the IMACS survey volume of $1.1 \times 10^{7} \mathrm{Mpc}^{3}$ noted earlier, we would expect $(1.0 \times$ $\left.10^{-4} h^{3} \mathrm{Mpc}^{-3} \mathrm{Gyr}^{-1}\right) \times(0.5 \mathrm{Gyr}) \times\left(1.1 \times 10^{7} \mathrm{Mpc}^{3}\right) \sim 190$ red-red pairs if our survey had placed a slit on every available galaxy and extracted redshifts with $100 \%$ completeness. As we have already shown, the two completeness correction factors encapsulated by Figures 2 and 3 can be used to map this expectation into an observed number of pairs in our survey. For red galaxies, our redshift completeness is $25 \%$ at $i^{\prime} \sim 22.5 \mathrm{mag}$, and as noted before, this should be squared to compute the pairwise completeness and then must be multiplied by the inverse of our sampling completeness. Therefore, our single detection of red-red close pair translates into $1 / 0.06 \times 10 \simeq 160$ pairs after applying the completeness correction factors, which

\footnotetext{
7 Note that the maximum separation of close pairs selected in the DEEP2 survey is $10 h^{-1} \mathrm{kpc}$ larger than the projected distance selection criterion used in this work.
}

is consistent with the number of predicted red-red pairs obtained from the DEEP2 survey.

Some of our conclusions can also be tested using published results from the VIMOS VLT Deep Survey (VVDS; de Ravel et al. 2009). These authors provide a set of 36 spectroscopically confirmed physical pairs with separations $r_{p}<20 h^{-1} \mathrm{kpc}$ and velocity differences $\Delta v<500 \mathrm{~km} \mathrm{~s}^{-1}$. The fraction of pairs corresponding to early-type galaxies is $\sim 8 \%$, which is significantly lower than the fraction $(25 \%)$ estimated when the pair criteria are expanded to separations up to $r_{p}=100 h^{-1} \mathrm{kpc}$. Evidently, most of the red-red pairs in the sample are seen at larger separations.

\subsection{Damp versus Dry Mergers}

Based on the analysis presented in the previous section, we conclude that there is a high degree of consistency in the predicted number of early-stage mergers (with their separations smaller than $20 h^{-1} \mathrm{kpc}$ ) seen in our pair analysis data, when compared with the published space densities for such systems. It is less clear that the late-stage mergers probed by morphological studies (e.g., that of Chou et al. 2011) are also consistent with these numbers, given the very high fraction $(>50 \%)$ of nearby late-stage mergers that are red.

Why are so many nearby late-stage mergers red? A possible solution might be the existence of a short-lived star formation epoch during the early merging stage. In this scenario, some galaxies in the field are neither truly "dry" nor truly "wet," since they still contain a small amount of gas (Rampazzo et al. 2005; Crocker et al. 2012). When two galaxies interact at the close-pair stage, a small amount of star formation is triggered by tides, which makes at least one of the galaxies appear blue. This nascent star formation period must be fairly transient, since during the late phase of the merger, the remnant must become red in a short period of time (of the order of the merging timescale, $\sim 0.5 \mathrm{Gyr}$ ). The result is a relatively high number density of dry mergers despite a paucity of red-red pairs. This begs the question: what combination of physics might result in weak tides that trigger star formation, along with other processes that truncate it? One possibility is a combination of tidally induced merging coupled with the hot halo picture, as discussed in Section 4.4 below. However, before describing this idea further, we must first consider the precise definition of a dry merger, both in our paper and in the literature.

In this context, it is interesting to consider how the restframe $\left(g^{\prime}-i^{\prime}\right)$ color-selection threshold we have used to define our red galaxy sample compares with the color-selection criteria adopted in other papers that also probe "red mergers" at intermediate redshifts. Our $\left(g^{\prime}-i^{\prime}\right)$ color threshold has relatively little sensitivity to contributions made by young stellar populations, so it is particularly interesting to compare our work to Lin et al. (2008, hereafter Lin08), who used a $(u-b)$ color threshold, and van Dokkum (2005, hereafter vD05), who used a $(b-r)$ color threshold, to define their red populations. To intercompare the surveys, we can use spectral synthesis models to investigate how much additional star formation is needed in order for an underlying old stellar population to no longer meet the red galaxy color-selection thresholds used in each of these papers. We used the BC03 code (Bruzual \& Charlot 2003) to construct SEDs comprised of a dominant very old stellar population polluted by star formation from a very young stellar population. The stellar mass of the template galaxy was fixed to a stellar mass of $5 \times 10^{10} M_{\odot}$. Both stellar populations were modeled using exponential star formation 
histories with an $e$-folding timescale of $1 \mathrm{Gyr}$ and a foregroundscreen dust extinction model with $\tau_{V}=1.0$. The ages for the old and the young stellar populations were fixed at $10 \mathrm{Gyr}$ and $0.1 \mathrm{Gyr}$, respectively, and we varied the stellar mass fraction between the old and the young populations before measuring the corresponding broadband colors. Our analysis shows that the $\left(g^{\prime}-i^{\prime}\right)$ color threshold used in the present paper is rather liberal-a $10 \%$ contribution to the total mass is needed from the young stellar population in order to exceed our color threshold. In contrast to this, the $(u-b)$ color threshold used by Lin08 can accommodate a $\sim 1 \%$ young stellar population, and the $(b-r)$ color threshold used by vD05 can essentially accommodate no young stellar population $(<1 \%)$. This also implies that these early-type galaxies may not be completely dry. While it is a mistake to assume that morphological characteristics uniquely map onto particular stellar populations, we suspect that our red galaxy sample is likely to be comprised of a combination of elliptical galaxies and early-type spirals, while the Lin08 and vD05 samples are probably dominated by pure ellipticals. And if the Lin08 and vD05 studies are of "dry mergers," then our study is mainly made of systems better described as "damp mergers." However, it is worth noting that a non-negligible fraction of the red galaxies presented in vD05 exhibit excesses in the 8 and $24 \mu \mathrm{m}$ bands (Desai et al. 2011), which suggests that at least some of these objects are star forming. Putative earlytype galaxies may appear to be almost dry using rest-frame visible color-selection criteria, but they may not be completely dry and/or may be undergoing gas-rich minor mergers (Desai et al. 2011). The present paper shows that even damp mergers are quite hard to find, so the truly dry mergers must be remarkably rare.

\subsection{Implications for the Hot Halo Model}

We conclude this section by noting the constraints placed on the hot halo quenching model by our results. As noted in the Introduction, in the hot halo quenching model, systems with halo masses greater than $10^{12} M_{\odot}$ shock-heat in-falling gas within the virial radius so that star formation is quenched. We have estimated the halo masses of all physical pairs, and are able to subdivide our physical pair sample into two groups, with halo mass greater than and less than $10^{12} M_{\odot}$, and denote these with different symbols in Figures 4 and 5 . These figures show no significant evidence for an enhancement in the fraction of red pairs with their projected separations smaller than $20 h^{-1} \mathrm{kpc}$ in the high-mass group. Indeed, as noted earlier, red-red pairs are simply rare at all masses, and there is an excess of blue systems, again at all masses, relative to the field. Rather than being quenched, it seems that star formation is enhanced by galaxy-galaxy interaction at the close-pair (early-stage merger) phase. This begs the following question: why is there so little evidence for shock-induced star formation quenching in our data, given the abundance of red late-stage mergers reported by Chou et al. (2011)? It is possible that the quantity of gas flooding into a potential well in a merger is sufficiently large so that shocks are inefficient until the merger is nearly complete. The crossing timescale of a close pair is only $\sim 60$ million years, and it may be difficult to increase the gas temperature in such a short time period by gravitational heating. This admittedly rather vague suggestion does hint at the possibility that timescales do matter, so that the degree of shock-induced quenching might depend strongly on the phase of the merger being witnessed. Testing this intriguing idea will require a much larger sample than that used in the present paper.
Another possible explanation is that the gas in these interacting galaxies is not falling into the pair's dark matter halo; instead, it is already present in the halo, and is quite likely being accreted onto the central regions of one or both galaxies, as predicted by simulations (Mihos \& Hernquist 1994; Di Matteo et al. 2007) and seen observationally (Barton et al. 2000; Ellison et al. 2011).

\section{CONCLUSIONS}

We have obtained spectra for $\sim 2800$ candidate close-pair galaxies at $0.1<z<1.2$ identified from the CFHTLS fields. Spectra of these systems were obtained using the multi-object spectrograph IMACS on the $6.5 \mathrm{~m}$ Magellan and DBSP on the $5 \mathrm{~m}$ Hale telescopes. These data allow us to constrain the rate of dry mergers at intermediate redshifts and to test the "hot halo" model for the quenching of star formation. Redshifts were obtained for $\sim 50 \%$ of the galaxies in our sample (1385 galaxies), and $\sim 80 \%$ of the redshift measurements (1115 galaxies) have confidence levels greater than unity. Because confirmation of physical pairs requires successful high-confidence redshifts for both galaxies in the pair, in the end we are left with 148 closepair candidates (296 galaxies). We used virial radii estimated from the correlation between dynamical and stellar masses published by Leauthaud et al. (2011) as a reference to select physically merging systems from this sample, based on halo occupation. We find that around $1 / 5$ of our candidate pairs (31 pairs) are physically associated and share a common dark matter halo. These pairs are divided into red-red, blue-red, and blue-blue systems using rest-frame $g^{\prime}-i^{\prime}$ colors, using the classification method introduced in Chou et al. (2011). After correcting for known selection effects, the fraction of blue-blue pairs is significantly greater than that of red-red and blue-red pairs. Given a fairly liberal rest-frame color-selection criterion in selecting red galaxies, red-red pairs are almost entirely absent from our sample, suggesting that red early-phase mergers are rare at $z \sim 0.5$. This result is consistent with that obtained from the DEEP2 survey (Lin et al. 2008). Our data support models with a short merging timescale $(<0.5 \mathrm{Gyr})$ in which star formation is enhanced in the early phase of mergers, but quenched in the late phase of mergers. Hot halo models may explain this behavior if the virial shocks that heat gas are inefficient until the merger is nearly complete.

\section{REFERENCES}

Abraham, R. G., Glazebrook, K., McCarthy, P. J., et al. 2004, AJ, 127, 2455

Abraham, R. G., Nair, P., McCarthy, P. J., et al. 2007, ApJ, 669, 184

Baldry, I. K., Glazebrook, K., Brinkmann, J., et al. 2004, ApJ, 600, 681

Balogh, M. L., Baldry, I. K., Nichol, R., et al. 2004, ApJ, 615, L101

Barton, E. J., Geller, M. J., \& Kenyon, S. J. 2000, ApJ, 530, 660

Bell, E. F., Wolf, C., Meisenheimer, K., et al. 2004, ApJ, 608, 752

Bertin, E., \& Arnouts, S. 1996, A\&AS, 117, 393

Birnboim, Y., \& Dekel, A. 2003, MNRAS, 345, 349

Birnboim, Y., Dekel, A., \& Neistein, E. 2007, MNRAS, 380, 339

Blanton, M. R. 2006, ApJ, 648, 268

Blumenthal, G. R., Faber, S. M., Primack, J. R., \& Rees, M. J. 1984, Nature, 311,517

Bohlin, R. C., Colina, L., \& Finley, D. S. 1995, AJ, 110, 1316

Bolzonella, M., Miralles, J., \& Pelló, R. 2000, A\&A, 363, 476

Bournaud, F., Dekel, A., Teyssier, R., et al. 2011, ApJ, 741, L33

Brammer, G. B., Whitaker, K. E., van Dokkum, P. G., et al. 2011, ApJ, 739, 24

Bridge, C. R., Appleton, P. N., Conselice, C. J., et al. 2007, ApJ, 659, 931

Bridge, C. R., Carlberg, R. G., \& Sullivan, M. 2010, ApJ, 709, 1067

Brooks, A. M., Governato, F., Quinn, T., Brook, C. B., \& Wadsley, J. 2009, ApJ, 694, 396

Bruzual, G., \& Charlot, S. 2003, MNRAS, 344, 1000

Bundy, K., Scarlata, C., Carollo, C. M., et al. 2010, ApJ, 719, 1969 
Cattaneo, A., Dekel, A., Devriendt, J., Guiderdoni, B., \& Blaizot, J. 2006, MNRAS, 370, 1651

Ceverino, D., \& Klypin, A. 2009, ApJ, 695, 292

Chou, R. C. Y., Bridge, C. R., \& Abraham, R. G. 2011, AJ, 141, 87

Cole, S., Helly, J., Frenk, C. S., \& Parkinson, H. 2008, MNRAS, 383, 546

Conroy, C., Wechsler, R. H., \& Kravtsov, A. V. 2007, ApJ, 668, 826

Conselice, C. J. 2006, ApJ, 638, 686

Conselice, C. J., Bershady, M. A., Dickinson, M., \& Papovich, C. 2003, AJ, 126,1183

Conselice, C. J., Bershady, M. A., \& Jangren, A. 2000, ApJ, 529, 886

Conselice, C. J., Bluck, A. F. L., Buitrago, F., et al. 2011, MNRAS, 413, 80

Cowie, L. L., \& Barger, A. J. 2008, ApJ, 686, 72

Cox, T. J., Jonsson, P., Primack, J. R., \& Somerville, R. S. 2006, MNRAS, 373,1013

Crocker, A., Krips, M., Bureau, M., et al. 2012, MNRAS, 2291

Croton, D. J., Springel, V., White, S. D. M., et al. 2006, MNRAS, 365, 11

Dekel, A., \& Birnboim, Y. 2006, MNRAS, 368, 2

Dekel, A., Sari, R., \& Ceverino, D. 2009, ApJ, 703, 785

De Propris, R., Driver, S. P., Colless, M., et al. 2010, AJ, 139, 794

de Ravel, L., Le Fèvre, O., Tresse, L., et al. 2009, A\&A, 498, 379

Desai, V., Dey, A., Cohen, E., Le Floc'h, E., \& Soifer, B. T. 2011, ApJ, 730,130

de Vaucouleurs, G., de Vaucouleurs, A., Corwin, H. G., Jr., et al. 1991, Third Reference Catalogue of Bright Galaxies (New York: Springer)

Di Matteo, P., Combes, F., Melchior, A.-L., \& Semelin, B. 2007, A\&A, 468, 61

Di Matteo, T., Springel, V., \& Hernquist, L. 2005, Nature, 433, 604

Eggen, O. J., Lynden-Bell, D., \& Sandage, A. R. 1962, ApJ, 136, 748

Ellison, S. L., Patton, D. R., Mendel, J. T., \& Scudder, J. M. 2011, MNRAS, 418, 2043

Faber, S. M., Willmer, C. N. A., Wolf, C., et al. 2007, ApJ, 665, 265

Fioc, M., \& Rocca-Volmerange, B. 1997, A\&A, 326, 950

Gabor, J. M., \& Davé, R. 2012, arXiv:1202.5315

Gabor, J. M., Davé, R., Finlator, K., \& Oppenheimer, B. D. 2010, MNRAS, 407, 749

Giodini, S., Pierini, D., Finoguenov, A., et al. 2009, ApJ, 703, 982

Gonzalez, A. H., Zaritsky, D., \& Zabludoff, A. I. 2007, ApJ, 666, 147

Hamuy, M., Suntzeff, N. B., Heathcote, S. R., et al. 1994, PASP, 106, 566

Hamuy, M., Walker, A. R., Suntzeff, N. B., et al. 1992, PASP, 104, 533

Heymans, C., Bell, E. F., Rix, H.-W., et al. 2006, MNRAS, 371, L60

Hopkins, P. F., Bundy, K., Croton, D., et al. 2010, ApJ, 715, 202

Hopkins, P. F., \& Hernquist, L. 2006, ApJS, 166, 1

Hopkins, P. F., Somerville, R. S., Hernquist, L., et al. 2006, ApJ, 652, 864

Hughes, T. M., \& Cortese, L. 2009, MNRAS, 396, L41
Jogee, S., Miller, S. H., Penner, K., et al. 2009, ApJ, 697, 1971

Karachentsev, I. D., Karachentseva, V. E., Huchtmeier, W. K., \& Makarov, D. I. 2004, AJ, 127, 2031

Kauffmann, G., Heckman, T. M., White, S. D. M., et al. 2003, MNRAS, 341,33

Kereš, D., Katz, N., Fardal, M., Davé, R., \& Weinberg, D. H. 2009, MNRAS, 395,160

Lambas, D. G., Alonso, S., Mesa, V., \& O’Mill, A. L. 2012, A\&A, 539, A45

Leauthaud, A., Finoguenov, A., Kneib, J.-P., et al. 2010, ApJ, 709, 97

Leauthaud, A., George, M. R., Behroozi, P. S., et al. 2011, ApJ, 744, L159

Le Borgne, D., \& Rocca-Volmerange, B. 2002, A\&A, 386, 446

Le Borgne, D., Rocca-Volmerange, B., Prugniel, P., et al. 2004, A\&A, 425, 881

Lilly, S. J., Le Fèvre, O., Renzini, A., et al. 2007, ApJS, 172, 70

Lin, L., Koo, D. C., Willmer, C. N. A., et al. 2004a, ApJ, 617, L9

Lin, L., Patton, D. R., Koo, D. C., et al. 2008, ApJ, 681, 232

Lin, Y.-T., Mohr, J. J., \& Stanford, S. A. 2004b, ApJ, 610, 745

López-Sanjuan, C., Balcells, M., Pérez-González, P. G., et al. 2010, A\&A, 518, A20

Lotz, J. M., Jonsson, P., Cox, T. J., \& Primack, J. R. 2008, MNRAS, 391, 1137

Lotz, J. M., Jonsson, P., Cox, T. J., \& Primack, J. R. 2010, MNRAS, 404, 575

Mandelbaum, R., Seljak, U., Kauffmann, G., Hirata, C. M., \& Brinkmann, J. 2006, MNRAS, 368, 715

Martin, D. C., Wyder, T. K., Schiminovich, D., et al. 2007, ApJS, 173, 342

Mihos, J. C., \& Hernquist, L. 1994, ApJ, 431, L9

Neistein, E., \& Dekel, A. 2008, MNRAS, 388, 1792

Panuzzo, P., Rampazzo, R., Bressan, A., et al. 2011, A\&A, 528, A10

Patton, D. R., Carlberg, R. G., Marzke, R. O., et al. 2000, ApJ, 536, 153

Pozzetti, L., Bolzonella, M., Zucca, E., et al. 2010, A\&A, 523, A13

Rampazzo, R., Annibali, F., Bressan, A., et al. 2005, A\&A, 433, 497

Rubin, V. C., Burstein, D., Ford, W. K., Jr., \& Thonnard, N. 1985, ApJ, 289,81

Scoville, N., Abraham, R. G., Aussel, H., et al. 2007, ApJS, 172, 38

Shapiro, K. L., Falcón-Barroso, J., van de Ven, G., et al. 2010, MNRAS, 402, 2140

Spitler, L. R., Forbes, D. A., Strader, J., Brodie, J. P., \& Gallagher, J. S. 2008, MNRAS, 385, 361

Springel, V., Di Matteo, T., \& Hernquist, L. 2005, ApJ, 620, L79

Sullivan, M., Le Borgne, D., Pritchet, C. J., et al. 2006, ApJ, 648, 868

Toomre, A. 1977, in Evolution of Galaxies and Stellar Populations, ed. B. M.

Tinsley \& R. B. G. Larson (New Haven, CT: Yale Univ. Observatory), 401

Toomre, A., \& Toomre, J. 1972, ApJ, 178, 623

van Dokkum, P. G. 2005, AJ, 130, 2647

Willmer, C. N. A., Faber, S. M., Koo, D. C., et al. 2006, ApJ, 647, 853 